\title{
Angiotensin receptor-neprilysin inhibition by sacubitril/valsartan attenuates doxorubicin-induced cardiotoxicity in a pretreatment mice model by interfering with oxidative stress, inflammation, and Caspase 3 apoptotic pathway
}

\author{
Ferhat Dindaş (D), Hüseyin Güngör* (D), Mehmet Ekici** (D), Pınar Akokay¹ (D), Füsun Erhan*** (D), \\ Mustafa Doğduş (i), Mehmet Birhan Yılmaz² (i)
}

Department of Cardiology, Uşak Training and Research Hospital; Uşak-Turkey

Departments of *Pharmacology, and **Physiology, ***Histology, Faculty of Veterinary, Cumhuriyet University; Sivas-Turkey

${ }^{1}$ Department of Histology, İzmir Kavram Vocational School; İzmir-Turkey

${ }^{2}$ Department of Cardiology, Faculty of Medicine, Dokuz Eylül University; İzmir-Turkey

\section{ABSTRACT}

Objective: Doxorubicin (DOX) is a well-known cardiotoxic agent, whereas sacubitril/valsartan (Sac/Val) is an effective treatment option in heart failure. In this study, we aimed to evaluate the effect of Sac/Val on DOX-induced cardiotoxicity in pretreatment mice model.

Methods: A total of 24 mice were equally classified into 4 groups; control group, DOX (20 mg/kg; fifth day), Sac/Val (80 mg/kg), and Sac/Val+DOX (Sac/Val was given from day one of the study before doxorubicin administration). Electrocardiography parameters, including durations of QRS, ST, OT, PP segment, and QT/PO index were measured. Total antioxidant status (TAS), total oxidant status (TOS), tumor necrosis factor- $\alpha$ (TNF- $\alpha$ ), interleukin $1 \beta$ (IL-1 $\beta)$, IL-6, NT-proBNP concentrations, and Caspase 3 activity were evaluated.

Results: At the end of the 9-day study duration, QRS, ST, OT intervals, QT/PO index and TAS, TOS, TNF- $\alpha$, IL-1 $\beta$, IL- 6 levels were significantly higher in the DOX group than in the control group $(\mathrm{p}<0.001)$. Moreover, there were significant differences only in the PP interval when comparing the Sac/Val+DOX and control groups (p<0.001). QRS, ST, OT intervals, and QT/PO index, TAS, TOS, TNF- $\alpha$, IL-1 $1 \beta$, IL-6 levels were significantly lower in the Sac/Val+ DOX group compared with the DOX group $(p<0.001)$. Furthermore, NT-proBNP levels were lower in the Sac/Val+DOX group compared with the DOX group along with less Caspase 3 apoptosis.

Conclusion: Sac/Val seems to be cardioprotective against DOX-induced cardiotoxicity in pretreatment mice model. These findings can be attributed to the antiarrhythmic, anti-inflammatory, antioxidant, and antiapoptotic effects of Sac/Val as shown in this study.

Keywords: sacubitril, valsartan, doxorubicin, cardiotoxicity, heart failure

Cite this article as: Dindaş F, Güngör H, Ekici M, Akokay P, Erhan F, Doğduş $M$, et al. Angiotensin receptor-neprilysin inhibition by sacubitril/valsartan attenuates doxorubicin-induced cardiotoxicity in a pretreatment mice model by interfering with oxidative stress, inflammation, and Caspase 3 apoptotic pathway. Anatol $J$ Cardiol 2021; 25: 821-8.

\section{Introduction}

Cancer is the most common cause of mortality worldwide after circulatory system diseases (1). Cancer is not the only cause of death in patients with cancer. The side effects of cancer therapy lead to an increase in the frequency of cardio- vascular diseases or the acceleration of the existing pathobiology (2). In the last decades, the development of treatment modalities, increase in screening methods, and the emergence of cardio-oncology have reduced such undesirable consequences (3). However, cardiotoxicity, which is defined as functional or structural impairment of the myocardium

Address for Correspondence: Dr. Ferhat Dindaş, Uşak Eğitim ve Araştırma Hastanesi,

Kardiyoloji Kliniği, Uşak-Türkiye

Phone: +90506 8756700 E-mail: frhtys@hotmail.com

Accepted Date: 16.07.2021 Available Online Date: 19.10.2021

(C) Copyright 2021 by Turkish Society of Cardiology - Available online at www.anatoljcardiol.com DOI:10.5152/AnatolJCardiol.2021.356 


\section{HIGHLIGHTS}

- Anthracycline group chemotherapeutics are indispensable in many tumor treatments.

- Reducing the cardiotoxic effects of anthracyclines is an important issue in cardio-oncology. A pretreatment strategy is recommended to avoid cardiotoxic effects.

- The main finding of this study was that sacubitril/valsartan, an angiotensin receptor-neprilysin inhibitor, was protected from DOX-induced cardiotoxicity in a pretreatment mouse model.

caused by anthracyclic group drugs, is one of the most common problems (4).

In the anthracycline group, cardiotoxicity occurs in 1 of 4 patients receiving doxorubicin (DOX), and the initial performance of the myocardium cannot be achieved with the current standard cardioprotective therapy in approximately half of these patients $(4,5)$. The main mechanism of DOX-induced cardiotoxicity is currently known as the accumulation of reactive oxygen species and inflammatory cytokines, blocking the antioxidative mechanism and increasing apoptosis in cardiocytes $(6,7)$. Molecular evidence of cardiotoxic insults has been associated with an increase in angiotensin II type 1 (AT1) and natriuretic peptide levels in embryogenetic animal studies $(8,9)$. Sac/Val is a crystalline compound formed by the combination of neprilysin inhibitor prodrug sacubitril and angiotensin receptor blocker valsartan (10). Both animal and clinical studies have shown that Sac/Val as secondary or tertiary care is beneficial, especially in clinically overt heart failure $(10,11)$. However, the European Society of Medical Oncology published that the use of prophylactic therapies with cardioprotective agents may be considered in patients with normal left ventricular ejection fraction (LVEF) and cardiovascular risk factors who are scheduled to undergo anticancer therapy with known cardiotoxic agents (3).

Of note, to the best of our knowledge, there is no study describing the potential prophylactic effect of Sac/Val. In this study, we tested the hypothesis that Sac/Val prophylactic treatment could protect cardiac function in DOX-induced cardiomyopathy pretreatment mice model.

\section{Methods}

\section{Drugs and chemicals}

Doxorubicin hydrochloride was purchased from Kocak (İstanbul, Turkey) and sacubitril/valsartan complex from Novartis (İstanbul, Turkey). Murine total antioxidant status (TAS), total oxidant status (TOS), tumor necrosis factor- $\alpha$ (TNF $\alpha$ ), interleukin $1 \beta$ (IL-1 $\beta$ ), and IL-6 (Shanghai Sunred Biological Technology Co. Ltd., Shanghai, China), N-terminal pro-B type natriuretic peptide (NT Pro-BNP) (Sinogeneclon Co. Ltd., Hangzhou, China) enzyme-linked immunosorbent assay (ELISA) kits were used. All other chemical agents were of analytical purity.

\section{Experimental protocols and ethical plan}

Balb-c mice (4-6 weeks old/18-20 g) were acclimated to their environment for 1 week before any study procedures. Animals were kept on a 12-hour light/12-hour dark cycle at $22 \pm 2^{\circ} \mathrm{C}$ with a relative humidity of $60 \% \pm 5 \%$. The mice had free access to food and water after acclimating to laboratory conditions for 1 week. Twenty-four mice were randomly divided into groups $(n=6)$ as shown in Table 1; the control group (CONTROL), $\mathrm{Sac} / \mathrm{Val}$ group (Sac/Val), Dox group (DOX), and Sac/Val+Dox group (Sac/Nal+DOX). The mice in the CONTROL and DOX groups were administered an equal volume of perioral $0.9 \%$ saline $(2.5$ $\mathrm{mL} / \mathrm{kg}$ ) for 9 days. On the fifth day in the CONTROL group, one hour after the perioral saline administration, intraperitoneal (IP) saline $(20 \mathrm{mg} / \mathrm{kg})$ was administered. In the DOX group, one hour after the perioral saline administration, single dose IP DOX (20 $\mathrm{mg} / \mathrm{kg}$ ) was administered (12). The mice in the Sac/Val+DOX and $\mathrm{Sac} / \mathrm{Val}$ groups were administered perioral Sac/Val $(80 \mathrm{mg} / \mathrm{kg}$ sacubitril+valsartan $1 / 1$ complex) in $0.9 \%$ saline by gavage for 9 days. On the fifth day, in the Sac/Val+DOX group, one hour after perioral Sac/Val administration, single dose of IP doxorubicin (20 $\mathrm{mg} / \mathrm{kg}$ ) was administered (13-15). In addition, $0.9 \%$ perioral saline was administered to the CONTROL and DOX groups to check for any potential effects of oral gavaging, and $0.9 \%$ IP saline was administered to CONTROL and Sac/Val groups to check for any potential effect of IP injection. All the analyses were performed at the end of 9 days. The study was conducted in accordance with the guidelines of the Declaration of Helsinki. All the animals were used in accordance with protocols and policies approved by the Local Institutional Animal Care and Use Committee and in accordance with all provisions of the Public Health Service Policy on humane care and use of laboratory animals (Approval No: 65202830-050.04.04-418, Date: 2020/06/25).

\begin{tabular}{|c|c|c|c|}
\hline Groups & 1-4,h day & $5^{\text {th }}$ day & 6-9th day \\
\hline \multirow[t]{2}{*}{ Control } & Saline (PO) & Saline (P0) & Saline (PO) \\
\hline & & Saline (P0) & \\
\hline \multirow[t]{2}{*}{$\mathrm{Sac} / \mathrm{Val}$} & Sac/Val (PO) & Sac/Val (PO) & Sac/Val (PO) \\
\hline & & Saline (IP) & \\
\hline \multirow[t]{2}{*}{ Dox } & Saline (PO) & Saline (P0) & Saline (PO) \\
\hline & & Doxorubicin (IP) & \\
\hline \multicolumn{4}{|c|}{ Sac/Val+Dox } \\
\hline & Sac/Val (PO) & Sac/Val (PO) & Sac/Val (PO) \\
\hline & & Doxorubicin (IP) & \\
\hline
\end{tabular}




\section{Electrocardiography evaluation}

Electrocardiography (ECG) was obtained as previously defined (16). The mice were anesthetized by xylazine hydrochloride $(5 \mathrm{mg} / \mathrm{kg} \mathrm{IP}$ ) and ketamine hydrochloride $(50 \mathrm{mg} / \mathrm{kg} \mathrm{IP})$. ECG records were obtained on conscious animals at the end of 9 days (Televet II Kruuse, Heusenstamn, Germany). The extremities of the mice were cleaned with alcohol to protect the flow of electricity. ECGs were recorded for derivation II (DII) with alligator clips (Televet $100^{\circledR}$ ). All ECG papers were scanned, uploaded to the computer, magnified at a speed of $200 \mathrm{~mm} / \mathrm{sec}$ and $40 \mathrm{~mm} /$ $\mathrm{mV}$ electrical calibration, and measured with digital image processing software (Televet 100 ECG version 7.0.0). ECG parameters included PQ interval, PP interval, QT interval, QRS duration, ST interval, and QT/PO cardiomyopathy index $(16,17)$. ECG was recorded at least for 5 minutes to determine the aforementioned most reliable intervals in DOX-induced cardiotoxicity as previously described $(16,17)$.

\section{Measurement of oxidant-antioxidant, cytokines activity, and NT-proBNP level}

TAS, TOS, TNF- $\alpha$, IL- $1 \beta$, and IL- 6 activity content were determined using the commercial ELISA kits (Sunred, China). After cutting heart samples, weight was checked and phosphate buffered saline (PBS) pH 7.4 was added. Rapidly frozen liquid nitrogen maintained samples at $2^{\circ} \mathrm{C}-8^{\circ} \mathrm{C}$. Heart tissue homogenates were prepared according to the manufacturer's instructions and centrifuged for 20 minutes at a speed of 2000-3000 rpm. All the absorbances were determined with an ELISA reader at a wavelength of $450 \mathrm{~nm}$. TAS activity was expressed as $\mathrm{U} / \mathrm{g}$ tissue, and TOS activity was expressed as nmol/g tissue. TNF- $\alpha$, IL- $1 \beta$, and IL-6 levels were expressed pg/tissue. Blood samples were obtained from the hearts of mice under anesthesia (after 9 days). The samples were centrifuged at $3053 \mathrm{~g}$ for 5 min within 1 hour of collection. Serum was collected and stored at $-80^{\circ} \mathrm{C}$. Aliquots were used one time, not subjected to freeze-thaw cycles for testing. Serum NT-proBNP levels were measured (Microplate washer RT 2100-2600) according to the manufacturer's instructions with ELISA using a Mouse NT-pro BNP Kit (SG-30273, Sinogeneclon, China). NT-proBNP level was expressed as $\mathrm{pg} / \mathrm{mL}$.

\section{Histochemical analysis of the specimens}

After electrocardiographic examination, the mice were sacrificed by cervical dislocation. Heart tissues were fixed in $10 \%$ formol for 24-48 hours for histochemical examinations. Tissues were washed under a stream for 1 night to remove the fixative. The tissues were dehydrated for 20 minutes in $70 \%, 80 \%$, and $96 \%$ ethyl alcohol series respectively and kept in 4 different acetone series for 20 minutes. The tissues were exposed to 2 different xylene for 30 minutes each for transparency; and after immersion with paraffin, the tissues were embedded in hard paraffin blocks. Sections of $5 \mu$ thickness were cut using a rotary microtome (RM 2255, Leica). Examinations were performed in a blinded manner.

\section{Hematoxylin-eosin protocol}

The sections taken were kept in an oven at $60^{\circ} \mathrm{C}$ for one night for deparaffinization process. The first was then subjected to 3 different xylene for 20 minutes (in the oven), and the other 2 for 10 minutes. Sections were stained with hematoxylin (01562E, Surgipath, Bretton, Peter Borough, Cambridgeshire) for $10 \mathrm{~min}$ utes after rinsing with distilled water. Sections passed through serial absolute alcohol after staining were kept in xylene and then sealed with entellan (UN 1866, Merck, Darmstadt, Germany).

\section{Immunohistochemistry protocol}

The sections were first kept in a $60^{\circ} \mathrm{C}$ oven for one night and then subjected to 3 changes of xylol for 30 minutes for transparency. The tissues were treated with trypsin and washed 3 times with PBS. They were incubated for one hour with blocking solution (TA125-UB, Lab Vision, Fremont, CA, USA). Primary antibody was kept with mouse specific anti-Caspase 3 (Bs-0081R, Bioss, USA) at $+4^{\circ} \mathrm{C}$ for one night. The sections washed with PBS were then treated with diaminabenzidinamine (DAB) (Roche, Germany) to determine the visibility of the immunohistochemical reaction. After painting the sections washed with distilled water, they were closed with entellan (UN 1866, Merck, Darmstadt, Germany). Anti-Caspase 3 antibody staining was performed to determine the distribution of Caspase 3 molecules in heart cells. To determine the distribution of these molecules between groups, semi-quantitative measurements were made in the heart tissue. The scoring was done as follows; 0 for each data: No positive staining; +: Slightly positive staining; ++: Moderate positive staining; +++ : Strong positive staining.

\section{Statistical analysis}

Statistical analyses were performed with the Statistical Package for Social Sciences version 25 (IBM Corp., Armonk, NY, USA). All the data were expressed as mean \pm standard deviation. All the data were tested for normality using the ShapiroWilk normality test. One-way analysis of variance was applied, and Tukey post-hoc test was used for multiple comparisons. The Kruskal-Wallis variance analysis was used to evaluate the significance of the differences in immunohistochemical results of Caspase 3. The data of histochemical findings were presented as median [interquartile range (IQR)]. The Dunn-Bonferroni posthoc method was performed as a result of Kruskal-Wallis. P values $<0.05$ were considered statistically significant two-sided.

\section{Results}

\section{Effect of Sac/Val on electrocardiography parameters}

ECG parameters were measured as described previously and are presented in Figure 1 and Table 2. On the fifth day, DOX administration alone caused a significant increase in ORS duration (19.83 $\pm 0.75 \mathrm{~ms}$ vs. $11.50 \pm 0.62 \mathrm{~ms})$, ST interval $(42.17 \pm 1.72 \mathrm{~ms}$ vs. $25.17 \pm 1.11 \mathrm{~ms})$, OT interval $(62.50 \pm 2.00 \mathrm{~ms}$ vs. $36.67 \pm 1.33 \mathrm{~ms})$, and $\mathrm{QT} / \mathrm{PO}$ index (1.66 \pm 0.06 vs. $0.74 \pm 0.03)$ in the $\mathrm{DOX}$ group compared 


\begin{tabular}{|c|c|c|c|c|}
\hline Variables & Control & Sac/Val & Dox & Sac/Val+Dox \\
\hline ORS (ms) & $11.50 \pm 0.62$ & $12.33 \pm 0.81^{\#}$ & $19.83 \pm 0.75^{*}$ & $14.00 \pm 0.89^{\#}$ \\
\hline ST (ms) & $25.17 \pm 1.11$ & $26.33 \pm 0.80^{\#}$ & $42.17 \pm 1.72^{*}$ & $27.00 \pm 1.12^{\#}$ \\
\hline $\mathrm{OT}(\mathrm{ms})$ & $36.67 \pm 1.33$ & $38.00 \pm 1.03^{\#}$ & $62.50 \pm 2.00^{*}$ & $41.00 \pm 0.86^{\#}$ \\
\hline $\mathrm{PP}$ (ms) & $142.67 \pm 3.29$ & $135.33 \pm 2.11^{\#}$ & $20.33 \pm 1.20^{*}$ & $22.00 \pm 1.15^{*}$ \\
\hline $\mathrm{PQ}(\mathrm{ms})$ & $50.00 \pm 2.00$ & $52.67 \pm 0.84^{\#}$ & $37.67 \pm 1.74^{*}$ & $45.00 \pm 1.44 \&$ \\
\hline QT/PQ index & $0.74 \pm 0.03$ & $0.72 \pm 0.03^{\#}$ & $1.66 \pm 0.06^{*}$ & $0.92 \pm 0.04^{\#}$ \\
\hline \multicolumn{5}{|c|}{$\begin{array}{l}\text { aVariables are expressed as mean } \pm \text { standard deviation } \\
\mathrm{b}_{\mathrm{n}=24} \\
{ }^{*} P<0.001 \text { compared with control } \\
\& P<0.05 \text { and } \# P<0.001 \text { compared with Dox } \\
\text { One-way ANOVA post-hoc Tukey } \\
\text { Dox - doxorubicin, ECG - electrocardiography; PP - PP interval, } P O \text { - PQ segment, ST - ST } \\
\text { interval, QRS - QRS duration, OT - QT interval, QT/PQ - QT interval divided by the PQ } \\
\text { segment, Sac/Val - sacubitril/valsartan }\end{array}$} \\
\hline
\end{tabular}

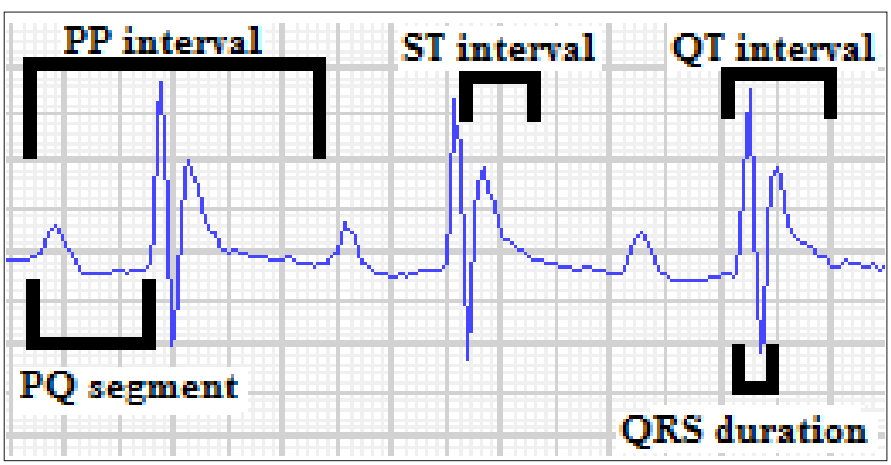

Figure 1. Quantitative evaluation of $P P$ interval, $P Q$ segment, $Q R S$ duration, ST interval, OT interval, and the cardiomyopathy index (QT/ PQ) from lead II tracings

with the CONTROL group ( $\mathrm{p}<0.001$ for all comparisons). In addition, in the Sac/Val+DOX group in which sacubitril/valsartan had been administered before DOX administration, QRS duration $(14.00 \pm 0.89$ $\mathrm{ms} ; 19.83 \pm 0.75 \mathrm{~ms})$, ST interval $(27.00 \pm 1.12 \mathrm{~ms} ; 42.17 \pm 1.72 \mathrm{~ms})$, OT interval $(41.00 \pm 0.86 \mathrm{~ms} ; 62.50 \pm 2.00 \mathrm{~ms})$, and $\mathrm{OT} / \mathrm{PQ}$ index $(0.92 \pm 0.04$; $1.66 \pm 0.06)$ were significantly lower than in the $\mathrm{DOX}$ group $(\mathrm{p}<0.001$ for all comparisons). The PP interval $(20.33 \pm 1.20 \mathrm{~ms} ; 142.67 \pm 3.29$ ms) was significantly shorter in the DOX group than in the CONTROL group $(p<0.001)$. Similarly, in the Sac/Nal+DOX group, the PP interval $(22.00 \pm 1.15 \mathrm{~ms}$ vs $142.67 \pm 3.29 \mathrm{~ms})$ was significantly shorter than in the CONTROL group ( $\mathrm{p}<0.001)$. In addition, $\mathrm{PO}$ segment (37.67 $\pm 1.74 \mathrm{~ms}$ vs. $50.00 \pm 2.00 \mathrm{~ms}$ ) were significantly shorter in DOX group than in the CONTROL group $(p<0.001)$. Furthermore, the PO interval $(45.00 \pm 1.44 \mathrm{~ms}$ vs. $50.00 \pm 2.00 \mathrm{~ms})$ in the Sac/ $\mathrm{Val}+\mathrm{DOX}$ group was significantly shorter than in the CONTROL group ( $p<0.05)$. ORS duration ( $12.33 \pm 0.81 \mathrm{~ms}$ vs. $19.83 \pm 0.75 \mathrm{~ms})$, OT interval $(38.00 \pm 1.03 \mathrm{~ms}$ vs. $62.50 \pm 2.00 \mathrm{~ms})$, ST interval $(26.33 \pm 0.80$ $\mathrm{ms}$ vs. $42.17 \pm 1.72 \mathrm{~ms})$, and $\mathrm{QT} / \mathrm{PQ}$ index $(0.72 \pm 0.03$ vs. $1.66 \pm 0.06)$ were shorter in the Sac/Val group than in the DOX group (for all $\mathrm{p}<0.001)$. However, in the Sac/Val group, the PP interval $(135.33 \pm 2.11$ $\mathrm{ms} ; 20.33 \pm 1.20 \mathrm{~ms}$ ) and $\mathrm{PQ}$ interval $(52.67 \pm 0.84 \mathrm{~ms} ; 37.67 \pm 1.74 \mathrm{~ms})$ were longer than in the DOX group (for all $p<0.001$ ).
Effect of Sac/Val on antioxidant-oxidant, cytokines activity, and NT-proBNP level

Biochemical parameters were presented in Table 3. TNF- $\alpha$ $(11735.81 \pm 317.66 \mathrm{pg} / \mathrm{g}$ vs. $6785.87 \pm 345.33 \mathrm{pg} / \mathrm{g}), \mathrm{IL}-1 \beta(735.62 \pm 47.45$ $\mathrm{pg} / \mathrm{g}$ vs. $321.22 \pm 11.29 \mathrm{pg} / \mathrm{g})$, IL-6 $(8696.60 \pm 293.88 \mathrm{pg} / \mathrm{g}$ vs. $4565.28 \pm 5990.51 \mathrm{pg} / \mathrm{g})$, and TOS $(308.93 \pm 23.88 \mathrm{nmol} / \mathrm{g}$; $155.04 \pm 16.00 \mathrm{nmol} / \mathrm{g}$ ) levels were significantly higher in DOX group compared with CONTROL group ( $\mathrm{p}<0.001$ for all comparisons). However, TAS $(167.73 \pm 8.85 \mathrm{U} / \mathrm{g} ; 302.42 \pm 10.27 \mathrm{U} / \mathrm{g}$ ) level was significantly lower in the DOX group mice than in the CONTROL group $(\mathrm{p}<0.001)$.

TNF- $\alpha(7636.36 \pm 438.07 \mathrm{pg} / \mathrm{g}$ vs. $11735,81 \pm 317,66 \mathrm{pg} / \mathrm{g}), \mathrm{IL}-1 \beta$ $(468.37 \pm 9.95 \mathrm{pg} / \mathrm{g}$ vs. $735.62 \pm 47.45 \mathrm{pg} / \mathrm{g}), \mathrm{IL}-6(5131.21 \pm 112.53$ $\mathrm{pg} / \mathrm{g}$ vs. $8696.60 \pm 293.88 \mathrm{pg} / \mathrm{g})$, and TOS $(195.87 \pm 3.82 \mathrm{nmol} / \mathrm{g}$ vs. $308.93 \pm 23.88 \mathrm{nmol} / \mathrm{g}$ ) levels were signific antly lower in the Sac/ $\mathrm{Val}+\mathrm{DOX}$ group than in the DOX group $(\mathrm{p}<0.001$ for all comparisons). In addition, TAS $(289.14 \pm 26.38 \mathrm{U} / \mathrm{g} ; 167.73 \pm 8.85 \mathrm{U} / \mathrm{g})$ level was significantly higher in the Sac/Val+DOX group than in the DOX group ( $p<0.001)$.

TNF- $\alpha(11735.81 \pm 317.66 \mathrm{pg} / \mathrm{g}$ vs. $6394.63 \pm 352.44 \mathrm{pg} / \mathrm{g}), \mathrm{IL}-1 \beta$ $(735.62 \pm 47.45 \mathrm{pg} / \mathrm{g}$ vs. $311.64 \pm 10.13 \mathrm{pg} / \mathrm{g})$, and TOS $(308.93 \pm 23.88$ $\mathrm{nmol} / \mathrm{g}$ vs. $151.97 \pm 8.57 \mathrm{nmol} / \mathrm{g}$ ) levels were significantly higher in the DOX group than in Sac/Val group $(p<0.001)$. Similarly, IL-6 level was significantly higher in the DOX group than in the Sac/ Val group $(8696.60 \pm 293.88 \mathrm{pg} / \mathrm{g}$ vs. $5990.51 \pm 787.82 \mathrm{pg} / \mathrm{g}$, respectively, $\mathrm{p}<0.01)$.

NT-proBNP $(403.61 \pm 57.35$ vs. $157.52 \pm 10.47 \mathrm{pg} / \mathrm{mL})$ levels were higher in the DOX group than in CONTROL group $(p<0.01)$. In the Sac/Val group, NT-proBNP level was lower than in the DOX group $(164.28 \pm 21.70$ vs. $403.61 \pm 57.35 \mathrm{pg} / \mathrm{mL}$, respectively, $\mathrm{p}<0.001)$. Furthermore, in the $\mathrm{Sac} / \mathrm{Val}+\mathrm{DOX}$ group, NT-proBNP levels were significantly lower than in the DOX group $(205.20 \pm 39.85$ vs. $403.61 \pm 57.35 \mathrm{pg} / \mathrm{mL}$, respectively, $\mathrm{p}<0.05)$.

\section{Histochemical findings}

Histochemical results are presented in Figure 2 and Table 4. Heart muscle cells and centrally located cell nuclei had a normal structure on examining the hematoxylin-eosin stained sections of the CONTROL group and the experimental group to which Sac/ Val was administered. In the DOX group, areas of irregularity, proteinaceous material accumulation, and hyperemia were detected between the muscle fibers. It was observed that degenerative changes decreased and streaking in cardiomyocytes was normal in the experimental group in which Dox was administered after Sac/Val. In the immunohistochemical Caspase 3 analysis, no immunoreactivity was observed in the CONTROL and the Sac/Val groups. Strong and intense Caspase 3 immunoreactivity was observed in the treatment group that received only DOX. However, Caspase 3 immunoreactivity was significantly decreased in cardiomyocytes in the group that received Sac/Val treatment before DOX. According to the semiquantitative histological score, Caspase 3 immunoreactivity was significantly lower in the Sac/Val+DOX group than in the DOX 


\begin{tabular}{|c|c|c|c|c|}
\hline Variables & Control & Sac/Val & Dox & Sac/Val+Dox \\
\hline TNF- $\alpha(p g / g$ tissue) & $6785.87 \pm 345.33$ & $6394.63 \pm 352.44^{\#}$ & $11735.81 \pm 317.66^{*}$ & $7636.36 \pm 438.07^{\#}$ \\
\hline IL-6 (pg/g tissue) & $4565.28 \pm 5990.51$ & $5990.51 \pm 787.82^{\&}$ & $8696.60 \pm 293.88^{*}$ & $5131.21 \pm 112.53^{\#}$ \\
\hline TAS (U/g tissue) & $302.42 \pm 10.27$ & $296.36 \pm 9.02^{\#}$ & $167.73 \pm 8.85^{*}$ & $289.14 \pm 26.38^{\#}$ \\
\hline NT-proBNP (pg/mL) & $157.52 \pm 10.47$ & $164.28 \pm 21.70^{\#}$ & $403.61 \pm 57.35^{* *}$ & $205.20 \pm 39.85 \& \&$ \\
\hline \multicolumn{5}{|c|}{$\begin{array}{l}\text { aVariables are expressed as mean } \pm \text { standard deviation } \\
\mathrm{b}_{\mathrm{n}=24} \\
* P<0.001 \text { compared with control } \\
\& \& P<0.05, \& P<0.01 \text {, and } \# P<0.001 \text { compared with Dox } \\
\text { One-way ANOVA post-hoc Tukey } \\
\text { Dox- doxorubicin, IL - interleukin, NT-ProBNP - N-terminal pro-B type natriuretic peptide, Sac/Val - sacubitril/valsartan, TAS - total antioxidant status, TNF - tumor necrosis factor, TOS - } \\
\text { total oxidant status }\end{array}$} \\
\hline
\end{tabular}

Table 4. Histochemical findings of study groups ${ }^{a}, b$

\begin{tabular}{|c|c|c|c|c|}
\hline Variable & $\begin{array}{l}\text { Control } \\
\text { p (IOR) }\end{array}$ & $\begin{array}{l}\text { Sac/Val } \\
\mu(I Q R)\end{array}$ & $\begin{array}{l}\text { Dox } \\
\mu(I Q R)\end{array}$ & $\begin{array}{l}\text { Sac/Nal+Dox } \\
\mu \text { (IOR) }\end{array}$ \\
\hline Caspase 3 & $0.5(0-1)$ & $0.5(0-1)^{\# \#}$ & $3.0(2-3)^{* *}$ & $1.0(1-2)^{\#}$ \\
\hline \multicolumn{5}{|c|}{$\begin{array}{l}\text { aVariables are expressed as median (IQR) } \\
\mathrm{b}_{\mathrm{n}=24} \\
{ }^{*} P<0.01 \text { compared with control } \\
{ }^{\#} P<0.05,{ }^{\#} P<0.01 \text { compared with Dox group } \\
\text { IQR - interquartile range }\end{array}$} \\
\hline
\end{tabular}

group [1.0 (1-2) vs. $3.0(2-3), p=0.02]$. Caspase 3 immunoreactivity was higher in the DOX group than in the CONTROL group [3.0 (2-3) vs. 0.50 (0-1), $p<0.001]$. In Sac/Val group, Caspase 3 immunoreactivity was lower than in the DOX group [0.50 (0-1) vs. 3.0 $(2-3), p<0.01]$.

\section{Discussion}

As the anthracycline group chemotherapeutics are indispensable drugs in many tumors, the effectiveness of the new agents should be evaluated along with considerations to reduce their toxic effects. The main finding of this study was that Sac/ Val, an angiotensin receptor-neprilysin inhibitor, protected from DOX-induced cardiotoxicity in a pretreatment mice model. Arrhythmogenic changes, cytokine accumulation, increase in free radicals, natriuretic peptide levels, and histopathological myocardial toxicity were less apparent in mice receiving Sac/ Val prior to DOX treatment than mice receiving DOX alone. According to our literature review, this is the first study designating the protective/preventive effect of Sac/Val in DOXinduced cardiomyopathy pretreatment mice model rather than the well-established benefit of Sac/Val in heart failure.

Doxorubicin targets the nucleotide helix via topoisomerase 2 as well as killing cancer cells, causing the production of malfunctioning proteins in the intracellular signaling system (18). Altered signal proteins, structural degeneration in both ion channels, and the calcium-calmodulin complex leads to development of arrhythmia owing to impaired impulse connections (19). The arrhythmo- genic cardiomyopathic effect of DOX was based on previous studies that showed prolongation of the $\mathrm{OT}$ interval and increase in the $0 T / P Q$ index, resulting in life-threatening ventricular tachycardia and fibrillation (20). Another possible mechanism designated that DOX increased the angiotensin 1 receptor in embryonic heart cells (9). Sac/Val is a complex that reduces ventricular fibrosis by inhibiting the angiotensin 1 receptor and increases enkephalin as well as natriuretic peptides in the neurohumoral system $(21,22)$. In a study with rats, enkephalinase inhibitors were found to prevent adrenaline-induced arrhythmias (23). A recent view is that Sac/Val may be a new antiarrhythmic drug because of an increase in enkephalin (22). In previous clinical studies, it was obvious that $\mathrm{Sac} / \mathrm{Val}$ decreased sudden cardiac death rate in patients with heart failure, and it did this by reducing ventricular arrhythmias $(24,25)$. In our results, the DOX group mice had cardiac electrotoxicity in the form of significant prolongation of the ventricular electrical activation markers, namely ORS, QT, ST durations, and QT/PO index. However, there was no significant change in ventricular electrical activity in Sac/Val+DOX group, but there was a deterioration in the PP interval, which might be an indicator of atrial arrhythmias. Recent studies have shown that $\mathrm{Sac} / \mathrm{Val}$ improves ventricular arrhythmias, but there are conflicting results regarding atrial arrhythmias $(26,27)$. Our study showed that Sac/Val prevented the arrhythmic effects of DOX in a pretreatment mice model, but this benefit was limited to ECG signals related to ventricular arrhythmias.

Beside the action of DOX through topoisomerase, it creates reactive oxygen species (ROS) that directly damages DNA, causing single strand breaks, base mismatches, and point mutations (28). ROS production causes stimulation of cytotoxic mechanisms independent of nucleotide damage, especially as a result of lipid peroxidation (28). It is accepted that lipid peroxidation initiates the apothtotic process in myocytes as a result of increased oxidative stress and activation of molecular pathways (29). In a recent study, natriuretic peptides were found to increase superoxide dismutase activity in hypertensive rats and prevent target organ damage through the oxidative distress 


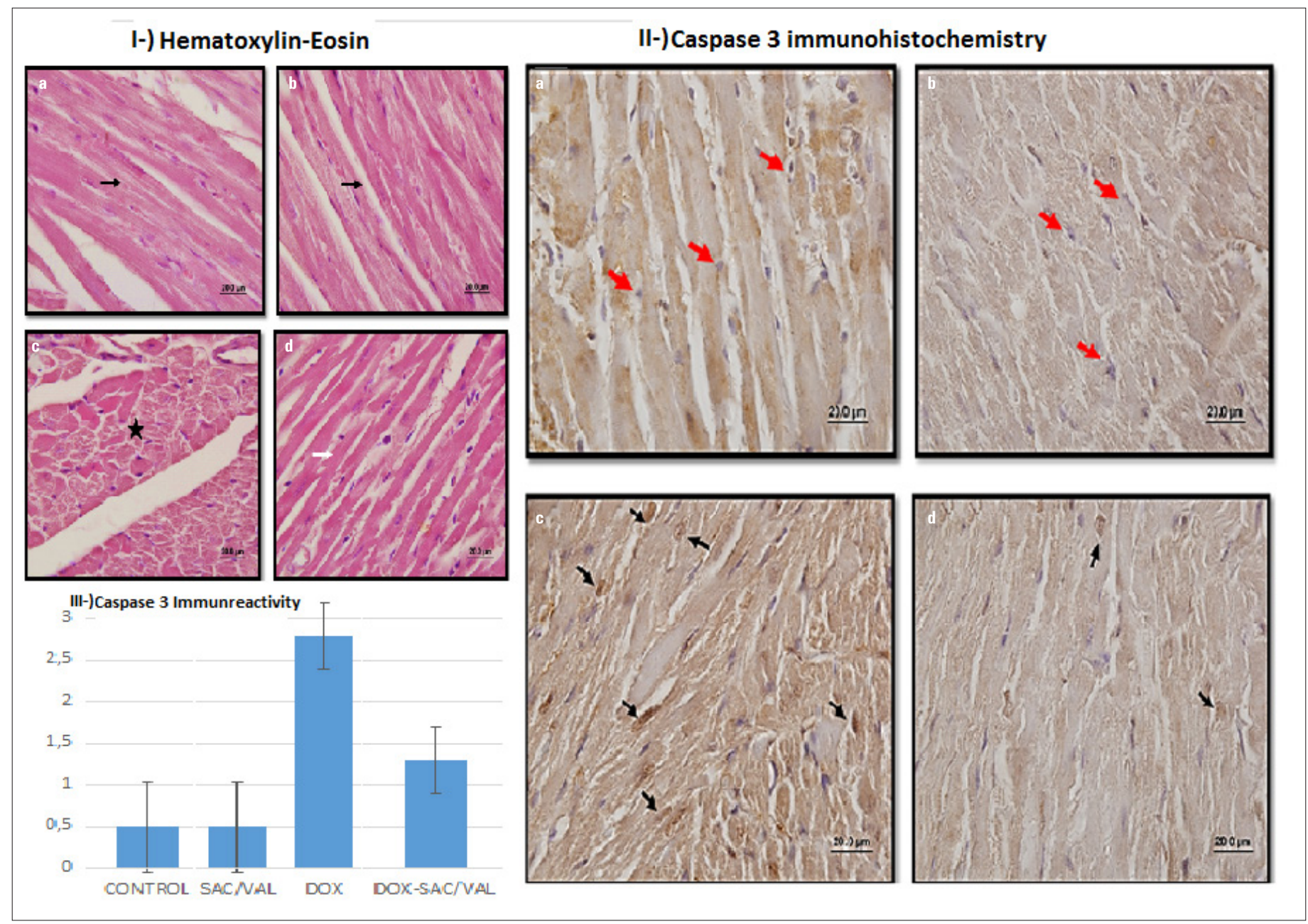

Figure 2. Histochemical analysis of groups. control (a), sac/val (b), dox(c), sac/val+dox(d). Fig. 2l. Hematoxylin-eosin (100×20 mm); normal histomorphology (black arrow) in cardiomyocytes in groups A and B. Proteinaceous material accumulation (black star) in group C. Normal histomorphological streaking (white arrow) in cardiomyocytes in group D. Fig. 2ll. Caspase 3 immunohistochemical analysis $(100 \times 20 \mathrm{~mm})$; no immunoreactivity was observed in cardiomyocytes (normal morphology-red arrows) in groups A and B. There was intense immune reactivity in the cardiomyocytes (black arrows) in group C. Immune reactivity in cardiomyocytes in group D was lower than in group C (decreased count of black arrows). Fig. 2III. Graph of mean \pm SD values of Caspase 3 immune reactivity

pathway (30). As Sac/Val provides natriuretic peptide optimization, we believe that it affected the antioxidant-oxidant balance positively. In our results, TAS and NT-proBNP levels were significantly higher in the group receiving DOX compared with the study group receiving prophylactic Sac/Val before DOX, whereas TOS level was the opposite.

A different cardiotoxic mechanism has been demonstrated in studies in that DOX increases proinflammatory cytokines containing IL sequences and TNF- $\alpha$ (31). Doxorubicin causes myocyte death by activating the caspase pathway by stimulating death receptors via TNF- $\alpha$ (7). Increased TNF- $\alpha$ and many interleukins cause free radical accumulation in the nitric oxide pathway (32). Stimulation of the continuous nitric oxide pathway results in insensitivity of intracellular calcium and contractile failure in myocytes (32). Moreover, increased TNF- $\alpha$, IL-1 $\beta$, and IL- 6 cause contractile defects in myocytes by changing the mitochondrial proteins rather than by disrupting the optimization of intracellular calcium $(33,34)$.
In post-treatment animal model studies, remodeling effect of Sac/ Val on cardiomyocyte was associated with mitochondrial fission proteins and the calcium calmodulin complex $(11,35)$. Accordingly, Sac/Val may have a different effect on mitochondrial proteins and calcium cellular metabolism as well as on inflammatory cytokines. In this study, it was shown that the inflammatory cytokines TNF- $\alpha$, IL-1 $\beta$, and IL-6 levels were lower in the group administered Sac/Val as a protective agent on DOX cardiomyopathy. The overall protective effects of Sac/Nal against DOX-induced cardiotoxicity might be linked to its effects on anti-cytokine capacity.

There is an interaction between DOX and various apoptotic pathways in the cardiomyocyte. For these pathways, caspase 3 activity may also be affected indirectly by calcineurin, cytochrome c, or directly by DOX (36). In addition, molecular studies have shown that the cardiotoxic effect of DOX was associated with mitochondrial dynamine related protein 1 (Drp1) protein, and this relationship has been demonstrated by caspase 3 activity (37). A 
recent study in a mouse heart failure model found that Sac/Nal complex administered after DOX treatment ameliorated mitochondrial dysfunction via Drp1 (11). We hypothesized that administration of Sac/Val before DOX treatment could prevent mitochondrial damage and alleviate cardiomyopathy. In our study, we found that prophylactic Sac/Val before DOX, which has direct or indirect effects on Caspase 3 activity, reduced the activation of the caspase 3 pathway. In clinical practice, it means less apoptotic pathway activation and more viable cardiomyocytes, though it remains to be proven by further studies. Nevertheless, it should be considered that prophylactic Sac/Val treatment applied before chemotherapy can provide significant benefits in terms of preventing the side effects of cancer treatment in the cardio-oncology area.

\section{Study limitations}

There were some limitations of our study. First, we did not perform echocardiography and hemodynamics examinations. Second, we studied a single but high-dose DOX model rather than a longterm DOX-administered cardiotoxicity model. Although complications are observed in the long-term anthracycline group in some patients, treatment-related cardiotoxicity occurs immediately after the first dose in many clinical patient groups (38). Finally, we did not assess mitochondrial function in cardiomyocytes.

\section{Conclusion}

It appears that prophylactic Sac/Val has certain protective/ preventive effects against DOX-induced cardiomyopathy in pretreatment mice model. Of note, cardiotoxic protective effects of Sac/Val against DOX can be attributed to its antiarrhythmic, anticytokine, antioxidant, and antiapoptotic effects as shown in this study. However, animal and large-scale clinical comparative studies of the protective efficacy of Sac/Val with other agents are needed in the field of cardio-oncology.

Acknowledgment: We would like to thank Nurullah Hatip for his valuable contributions.

Conflict of interest: None declared.

Peer-review: Externally peer-reviewed.

Author contributions: Concept - F.D., H.G., M.E.; Design - F.D., H.G., M.E.; Supervision - F.D., M.D., M.B.Y.; Fundings - None; Materials H.G., M.E., P.A., F.E.; Data collection \&/or processing - F.D., H.G., M.E.; Analysis \&/or interpretation - F.D., M.E., P.A., F.E., M.D.; Literature search - F.D., H.G., M.D.; Writing - F.D., M.D., M.B.Y; Critical review F.D., M.D., M.B.Y.

\section{References}

1. Ferlay J, Colombet M, Soerjomataram I, Mathers C, Parkin DM, Piñeros $\mathrm{M}$, et al. Estimating the global cancer incidence and mortality in 2018: GLOBOCAN sources and methods. Int J Cancer 2019; 144: 1941-53. [Crossref]
2. Armstrong GT, Oeffinger KC, Chen $Y$, Kawashima T, Yasui $Y$, Leisenring $\mathrm{W}$, et al. Modifiable risk factors and major cardiac events among adult survivors of childhood cancer. J Clin Oncol 2013; 31: 3673-80. [Crossref]

3. Curigliano G, Lenihan D, Fradley M, Ganatra S, Barac A, Blaes A, et al. Management of cardiac disease in cancer patients throughout oncological treatment: ESMO consensus recommendations. Ann Oncol 2020; 31: 171-90. [Crossref]

4. Yeh ET, Bickford CL. Cardiovascular complications of cancer therapy: incidence, pathogenesis, diagnosis, and management. J Am Coll Cardiol 2009; 53: 2231-47. [Crossref]

5. Cardinale D, Colombo A, Lamantia G, Colombo N, Civelli M, De Giacomi G, et al. Anthracycline-induced cardiomyopathy: clinical relevance and response to pharmacologic therapy. J Am Coll Cardiol 2010; 55: 213-20. [Crossref]

6. Wallace KB. Doxorubicin-induced cardiac mitochondrionopathy. Pharmacol Toxicol 2003; 93: 105-15. [Crossref]

7. Zhao L, Zhang B. Doxorubicin induces cardiotoxicity through upregulation of death receptors mediated apoptosis in cardiomyocytes. Sci Rep 2017; 7: 44735. [Crossref]

8. Koh E, Nakamura T, Takahashi H. Troponin-T and brain natriuretic peptide as predictors for adriamycin-induced cardiomyopathy in rats. Circ J 2004; 68: 163-7. [Crossref]

9. Huang CY, Chen JY, Kuo CH, Pai PY, Ho TJ, Chen TS, et al. Mitochondrial ROS-induced ERK1/2 activation and HSF2-mediated AT1 $\mathrm{R}$ upregulation are required for doxorubicin-induced cardiotoxicity. J Cell Physiol 2018; 233: 463-75. [Crossref]

10. McMurray JJ, Packer M, Desai AS, Gong J, Lefkowitz MP, Rizkala $A R$, et al.; PARADIGM-HF Investigators and Committees. Angiotensin-neprilysin inhibition versus enalapril in heart failure. $\mathrm{N}$ Engl J Med 2014; 371: 993-1004. [Crossref]

11. Xia Y, Chen Z, Chen A, Fu M, Dong Z, Hu K, et al. LCZ696 improves cardiac function via alleviating Drp1-mediated mitochondrial dysfunction in mice with doxorubicin-induced dilated cardiomyopathy. J Mol Cell Cardiol 2017; 108: 138-48. [Crossref]

12. Nozaki N, Shishido T, Takeishi Y, Kubota I. Modulation of doxorubicin-induced cardiac dysfunction in toll-like receptor-2-knockout mice. Circulation 2004; 110: 2869-74. [Crossref]

13. Aykan DA, Koca TT, Yaman S, Eser N. Angiotensin converting enzyme and neprilysin inhibition alter pain response in dexhamethasoneinduced hypertensive rats. Pharmacol Rep 2019; 71: 306-10. [Crossref]

14. Boutagy NE, Feher A, Pfau D, Liu Z, Guerrera NM, Freeburg LA, et al. Dual Angiotensin Receptor-Neprilysin Inhibition With Sacubitril/ Valsartan Attenuates Systolic Dysfunction in Experimental Doxorubicin-Induced Cardiotoxicity. JACC CardioOncol 2020; 2: 774-87. [Crossref]

15. Singal PK, Iliskovic N, Li T, Kumar D. Adriamycin cardiomyopathy: pathophysiology and prevention. FASEB J 1997; 11: 931-6. [Crossref]

16. Pereira JA, Mauricio AF, Marques MJ, Neto HS. Dual Therapy Deflazacort/Doxycyclyne Is Better Than Deflazacort Monotherapy to Alleviate Cardiomyopathy in Dystrophin-Deficient mdx Mice. J Cardiovasc Pharmacol Ther 2017; 22: 458-66. [Crossref]

17. Bostick B, Yue Y, Long C, Duan D. Prevention of dystrophindeficient cardiomyopathy in twenty-one-month-old carrier mice by mosaic dystrophin expression or complementary dystrophin/utrophin expression. Circ Res 2008; 102: 121-30. [Crossref]

18. Tewey KM, Rowe TC, Yang L, Halligan BD, Liu LF. Adriamycininduced DNA damage mediated by mammalian DNA topoisomerase II. Science 1984; 226: 466-8. [Crossref] 
19. Wagner S, Rokita AG, Anderson ME, Maier LS. Redox regulation of sodium and calcium handling. Antioxid Redox Signal 2013; 18: 106377. [Crossref]

20. Larsen RL, Jakacki RI, Vetter VL, Meadows AT, Silber JH, Barber G. Electrocardiographic changes and arrhythmias after cancer therapy in children and young adults. Am J Cardiol 1992; 70: 73-7. [Crossref]

21. Hubers SA, Brown NJ. Combined Angiotensin Receptor Antagonism and Neprilysin Inhibition. Circulation 2016; 133: 1115-24. [Crossref]

22. Sarrias A, Bayes-Genis A. Is Sacubitril/Valsartan (Also) an Antiarrhythmic Drug? Circulation 2018; 138: 551-3. [Crossref]

23. Lishmanov luB, Maslov LN, Uskina EV. Anti-arrhythmia effects of enkephalinase inhibitors. Eksp Klin Farmakol 2001; 64: 28-30. [Article in Russian]

24. de Diego C, González-Torres L, Núñez JM, Centurión Inda R, Martin-Langerwerf $D A$, Sangio $A D$, et al. Effects of angiotensinneprilysin inhibition compared to angiotensin inhibition on ventricular arrhythmias in reduced ejection fraction patients under continuous remote monitoring of implantable defibrillator devices. Heart Rhythm 2018; 15: 395-402. [Crossref]

25. Martens $P$, Nuyens $D$, Rivero-Ayerza $M$, Van Herendael $H$, Vercammen J, Ceyssens W, et al. Sacubitril/valsartan reduces ventricular arrhythmias in parallel with left ventricular reverse remodeling in heart failure with reduced ejection fraction. Clin Res Cardiol 2019; 108: 1074-82. [Crossref]

26. Li LY, Lou 0, Liu GZ, Lv JC, Yun FX, Li TK, et al. Sacubitril/valsartan attenuates atrial electrical and structural remodelling in a rabbit model of atrial fibrillation. Eur J Pharmacol 2020; 881: 173120. [Crossref]

27. Suo Y, Yuan M, Li H, Zhang Y, Li Y, Fu H, et al. Sacubitril/Valsartan Improves Left Atrial and Left Atrial Appendage Function in Patients With Atrial Fibrillation and in Pressure Overload-Induced Mice. Front Pharmacol 2019; 10: 1285. [Crossref]

28. Gewirtz DA. A critical evaluation of the mechanisms of action proposed for the antitumor effects of the anthracycline antibiotics adriamycin and daunorubicin. Biochem Pharmacol 1999; 57: 72741. [Crossref]
29. Minotti G, Menna P, Salvatorelli E, Cairo G, Gianni L. Anthracyclines: molecular advances and pharmacologic developments in antitumor activity and cardiotoxicity. Pharmacol Rev 2004; 56: 185-229. [Crossref]

30. Romero M, Caniffi C, Bouchet G, Costa MA, Elesgaray R, Arranz C, et al. Chronic treatment with atrial natriuretic peptide in spontaneously hypertensive rats: beneficial renal effects and sex differences. PLoS One 2015; 10: e0120362. [Crossref]

31. Pecoraro M, Del Pizzo M, Marzocco S, Sorrentino R, Ciccarelli M, laccarino $\mathrm{G}$, et al. Inflammatory mediators in a short-time mouse model of doxorubicin-induced cardiotoxicity. Toxicol Appl Pharmacol 2016; 293: 44-52. [Crossref]

32. Goldhaber JI, Kim KH, Natterson PD, Lawrence T, Yang P, Weiss JN. Effects of TNF-alpha on [Ca2+]i and contractility in isolated adult rabbit ventricular myocytes. Am J Physiol 1996; 271 (4 Pt 2): H144955. [Crossref]

33. Shen $Y L$, Shi $Y Z$, Chen GG, Wang $L L$, Zheng MZ, Jin HF, et al. TNF- $\alpha$ induces Drp1-mediated mitochondrial fragmentation during inflammatory cardiomyocyte injury. Int J Mol Med 2018; 41: 2317-27. [Crossref]

34. Xu S, Wang P, Zhang H, Gong G, Gutierrez Cortes N, Zhu W, et al. CaMKII induces permeability transition through Drp1 phosphorylation during chronic $\beta$-AR stimulation. Nat Commun 2016; 7: 13189. [Crossref]

35. Chang PC, Wo HT, Lee HL, Lin SF, Chu Y, Wen MS, et al. Sacubitril/ Valsartan Therapy Ameliorates Ventricular Tachyarrhythmia Inducibility in a Rabbit Myocardial Infarction Model. J Card Fail 2020; 26: 527-37. [Crossref]

36. Octavia Y, Tocchetti CG, Gabrielson KL, Janssens S, Crijns HJ, Moens AL. Doxorubicin-induced cardiomyopathy: from molecular mechanisms to therapeutic strategies. J Mol Cell Cardiol 2012; 52 : 1213-25. [Crossref]

37. Catanzaro MP, Weiner A, Kaminaris A, Li C, Cai F, Zhao F, et al. Doxorubicin-induced cardiomyocyte death is mediated by unchecked mitochondrial fission and mitophagy. FASEB J 2019; 33: 11096-108. [Crossref]

38. Doroshow JH. Anthracycline antibiotic-stimulated superoxide, hydrogen peroxide, and hydroxyl radical production by $\mathrm{NADH}$ dehydrogenase. Cancer Res 1983; 43: 4543-51. 